\title{
Size-dependent accumulation and toxicity of PS-MPs in earthworms (Eisenia foetida)
}

\author{
XUE XIAO $^{1}$, ERKAI HE $^{1 *}$, YETAO TANG ${ }^{1,2}$, RONGLIANG \\ $\mathrm{QIU}^{1,2,3}$
}

${ }^{1}$ School of Environmental Science and Engineering, Sun Yatsen University, Guangzhou 510006, China (correspondence: heerk@mail.sysu.edu.cn )

${ }^{2}$ Guangdong Provincial Key Lab for Environmental Pollution Control and Remediation Technology, Sun Yat-sen University, Guangzhou 510006, China (eestyt@mail.sysu.edu.cn)

${ }^{3}$ Guangdong Laboratory for Lingnan Modern Agriculture, South China Agriculture University, Guangzhou 510642, China (eesqrl@mail.sysu.edu.cn)

Microplastics are widely spread in terrestrial systems, while, at present, attention has been mostly focused on potential risk of microplastic in aquatic systems $[1,2]$. In this study, earthworms Eisenia fetida were exposed to polystyrene microplastics (PS-MPs) of different size (70 nm, $1 \mu \mathrm{m}$ and 10 $\mu \mathrm{m})$ and exposure dose $(0.5 \%, 5 \%$ and $10 \% \mathrm{w} / \mathrm{w}$ in food), aiming to achieve a comprehensive understanding of the accumulation and toxicological process of MPs.

Mortality, growth, oxidative stress and energy-metabolize were assessed to evaluate the toxicological effects of PS-MPs on E. fetida. Results showed that after 28 days of exposure, there was no significant influence of different PS-MPs treatments on mortality of earthworms. Organisms treated with $70 \mathrm{~nm}$ PS-MPs showed an obvious decrease of growth with increasing exposure dose. Compared to $1 \mu \mathrm{m}$ and $10 \mu \mathrm{m}$ PS-MPs treatments, $70 \mathrm{~nm}$ PS-MPs induced a more significant effect on the activities of peroxidase (POD), catalase (CAT), the content of malonaldehyde (MDA), and the concentration of ATP and non-esterified fatty acid (NFFA). These indicate that oxidative stress and energy influence are induced after exposure of MPs, especially under treatment of nano MPs. Furthermore, accumulation and distribution of PS-MPs in earthworms were analysed with laser confocal microscopy by exposing to fluorescent PS-MPs. Results showed different distribution patterns among PS-MPs of different size. Aggregation was observed for $70 \mathrm{~nm}$ and 1 $\mu \mathrm{m}$ PS-MPs in earthworm. The results of this study suggest that the size of MPs in terrestrial system is a key factor which should be considered for evaluating the toxicity of PS-MPs on soil organisms.

[1] Huerta Lwanga et al. (2016) Environ. Sci. Technol 50, 2685-2691. [2] Anderson Abel De Souza Machado et al. (2018) Glob Change Biol 24, 1405-1416. 